\title{
A Semiotic Approach to Integrative Negotiation
}

\author{
Janos J. Sarbo \\ ICIS, Radboud University Nijmegen, The Netherland \\ janos@cs.ru.nl
}

\begin{abstract}
An analysis of two-party integrative negotiation reveals the potential of this type of bargaining for being interpreted as a conceptualization process. Past research on a semiotic model of human processing shows the possibility of a definition of a uniform representation of knowledge. In this paper we suggest that by combining the above results integrative negotiation can be positioned as an abductive process. We justify our hypothesis by means of experimental data.
\end{abstract}

Keywords: Negotiation, Abduction, Peircean theory, Sign aspects, Cognitive model.

\section{Introduction}

Arguably the most efficient strategy for negotiation is win-win or two-party integrative negotiation [1], [10]. In this paper we are concerned with the question how negotiation efficiency can be increased through goal-driven communication between the parties. We show that win-win negotiation involves a process which is isomorphic to a Peircean model of cognitive activity [8]. An analysis of the latter process reveals types of information involved by its events. A combination of the above results enables the conclusion to be drawn that, in negotiation, if the parties get stuck at some stage, this could be because of a lack of information necessary for a realization of some event in the bargaining process. Communication of the required information may enable the parties to further develop their negotiation and bring it to a successful end, eventually.

We assume that, in order to get hold of the above problem of communication, the parties need to represent, besides their own information, a possible conceptualization of the negotiation process by the other party as well. This is where the Peircean model comes handy. By virtue of its categorical foundation, this model enables a uniform representation of knowledge in any domain (or, from any perspective of interpretation) hence a merging of representations from different perspectives by the parties, into a single representation. Through analyzing the events of the arising process during negotiation, on-line, the parties may derive information that can be helpful for the bargaining other party. This way of communication is akin to abduction, as it may enable to establish a relation between knowledge elements, which relation could not be derived by a party on the basis of his/her actual knowledge, neither in a deductive nor an inductive fashion. 
In order to enhance the efficiency of win-win negotiation, various off-line methods have been developed in the past. Examples are template design and evaluation, and algorithms for developing Pareto optimal solutions [5]. A problem shared by those methods is by virtue of their distant relation with the bargaining process.

Experience with a course on negotiation at Radboud University Nijmegen, The Netherlands, shows that the use of integrative negotiation is by far not obvious. Following an analysis of exercises in bargaining, students tend to stuck at some stage in their negotiation, keep repeating their earlier arguments hence make no progress. Unless a trained mediator is involved, integrative negotiation may not arise. In order to make the parties comply with that strategy, this course applies a technique that forces the parties to co-operate. ${ }^{1}$ This is achieved by means of setting a shared goal that both parties must agree on, and from which goal the aim of their negotiation necessarily follows. In a concrete example, a couple of students were asked to act as managers of a company. Following their instruction they had competing interests concerning the solution of a problem of the company. The shared goal was set by aiming at an increase of profit by the company. The parties were asked by a mediator (this role was played by a third student) if this goal does comply with their interest. As the answer must be positive (otherwise the managers were not able to correctly function anymore), the parties were 'primed' for acting in a co-operative fashion. Later when they were asked to develop new initiatives for a solution, they could not refuse inventing ideas that at least partly respected the interest of the negotiating other party as well.

Besides win-win bargaining, another example of integrative negotiation is problem elicitation. A distinguishing property of the latter is the existence of a spontaneous interest by the bargaining parties, for achieving a common goal. This may explain why the parties themselves may be able to generate helpful communication thereby enhancing their negotiation process [3]. In this paper we capitalize on the analogy between the two kinds of negotiation phenomena above and illustrate our approach with an analysis of a case study in problem elicitation. The development of a methodology enabling helpful communication is on our current agenda.

The structure of the paper is the following. We begin with an analysis of integrative negotiation from a process perspective (Sect. 2). This is followed by an introduction of a Peircean model of human information processing (Sect. 3), and how this model may enable a generation of efficient communication in win-win bargaining (Sect. 4). The proposed approach is justified by an analysis of experimental data (Sect. 5). We close the paper with a summary of results (Sect. 6).

\section{Integrative Negotiation as a Conceptualization Process}

Integrative negotiation, first introduced in [10], is a strategy that has the potential to achieve an agreement that gives everyone what they want and this way enables the parties to produce a more satisfactory output. This strategy is different from the one used by positional bargaining, which is based on fixed, opposing viewpoints (cf.

\footnotetext{
${ }^{1}$ Jan van den Broek (pers. comm., 2009).
} 
positions) and tends to result in a compromise or in no agreement at all. Compromises may not satisfy the true interest of the negotiating parties.

An oft-cited example of integrative negotiation and a creation of joint value is that of the dispute between two sisters over an orange [2]. Based on their positions, their mother cuts the orange in half and gives each girl one half. This outcome represents a compromise. However, if the mother was able to find out why the girls wanted the orange - what her interests were - there could have been a different, win-win outcome. This is because one girl wanted to eat the meat of the orange, but the other just wanted the peel to use in baking some cookies. If their mother had known their interests or were able to make the girls elicit their problem, they could have both gotten all of what they wanted, rather than just half.

According to [1], [4], [9], integrative negotiation can be characterized by a process, in which, first, each side's interest is sorted out. Their needs and why they want that is abstracted into possible demands. Next, they are asked to picture how their demands may be perceived by the other side, what may be standing in the way, which complementary information would enable an agreement, and in that context, how the interests of the two sides could be turned into actual demands. Integrative negotiation reaches its goal when those actual demands by the parties are combined into an agreement or a proposition of a solution of the input problem.

From the above analysis we draw the conclusion that integrative negotiation, as a process, makes use of four types of operation on the input problem appearing as a phenomenon. These are sorting, abstraction, complementation, and predication (proposition formation). In the next section we suggest that this process can be isomorphic to a cognitively based model of human processing. By making use of the potential analogy between the two models we show that integrative negotiation can be positioned as a conceptualization process. An advantage of the application of a cognitively based model is that it may give insight in the types of events of bargaining, and how the efficiency of those events can be increased by means of communication.

\section{$3 \quad$ Knowledge in Formation (KIF)}

The cognitively based model of human processing, introduced by our Knowledge in Formation (KIF) research project, has been presented at various ICISO conferences in the past [6], [11]. The used representation is based on the assumption, suggested by [12], that the aim of cognitive activity is the generation of a response on the input stimulus. A process model of response generation can be given as follows.

In a single interaction, the stimulus, appearing as an effect, is affecting the observer occurring in some state. The qualities of this state $\left(\mathrm{q}_{2}\right)$ and effect $\left(\mathrm{q}_{1}\right)$, which are in focus, and complementary memory knowledge or context $(C)$ triggered by $\mathrm{q}_{2}$ and $\mathrm{q}_{1}$, define the input for information processing $\left(\left[q_{2} q_{1} C\right]\right)$. See Fig. 1. The goal of this process is the generation of a relation, explaining why this effect is occurring to this state. In order to achieve this goal, the observer or interpreting system has to sort out the two types of qualities and context $\left(\left[\mathrm{q}_{2}\right],\left[\mathrm{q}_{1}\right],[\mathrm{C}]\right)$; abstract the input qualities in 
focus into independent collections $\left(\left(\mathrm{q}_{2}\right),\left(\mathrm{q}_{1}\right)\right)$; complete them with complementary knowledge by the interpreting system $\left(\left(\mathrm{q}_{2}, \mathrm{C}\right),\left(\mathrm{q}_{1}, \mathrm{C}\right)\right)$; and merge the obtained representations into a single relation $\left(\left(\mathrm{q}_{2}, \mathrm{C}\right)-\left(\mathrm{q}_{1}, \mathrm{C}\right)\right)$ through predication. We assume that the above process can be executed in cyclic fashion, recursively. Note that each one of the expressions generated by this process is a representation of an interaction between already existing input expressions interpreted as a state and an effect. For instance, $\left(\mathrm{q}_{2}\right)$ is a representation of the interaction between $\left[\mathrm{q}_{2}\right]$ (state) and $\left[\mathrm{q}_{1}\right]$ (effect) in the sense of relative difference hence an expression of the abstract input state irrespective of the input effect. Another example is $\left(\mathrm{q}_{2}, \mathrm{C}\right)$, which is a representation of the interaction between $\left(\mathrm{q}_{2}\right)$ (state) and $[\mathrm{C}]$ (effect) hence an expression of the abstract input state in context.

The four types of interactions are marked by their characteristic information. In sorting, this is the nature of quality, focus or complementary; in abstraction, the difference between $\left[\mathrm{q}_{2}\right]$ and $\left[\mathrm{q}_{1}\right]$; in complementation, the possible relation( $\mathrm{s}$ ) between $\mathrm{q}_{2}$ and $[\mathrm{C}]$, and between $\mathrm{q}_{1}$ and $[\mathrm{C}]$; in predication, the actual relation between $\left(\mathrm{q}_{2}, \mathrm{C}\right)$ and $\left(\mathrm{q}_{1}, \mathrm{C}\right)$. In our model of negotiation, in Sect. 4 , we assume that efficient communication may capitalize on the above informational classification of the interpretation events.

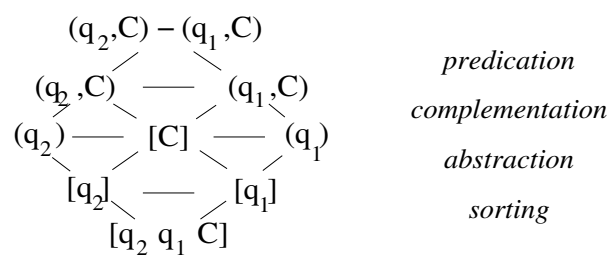

Fig. 1. The KIF process model of cognitive activity

State $\left(\mathrm{q}_{2}\right)$ and effect $\left(\mathrm{q}_{1}\right)$ stand for information in focus. Information, which is not in focus hence complementary, is represented by the context $(\mathrm{C})$. Horizontal lines are used to designate interactions between input representations (cf. positions in the processing schema). Square brackets indicate that an entity is not yet interpreted, usual bracket symbols indicate that some interpretation is already available. The types of interpretation events are displayed on the right-hand side, in italics.
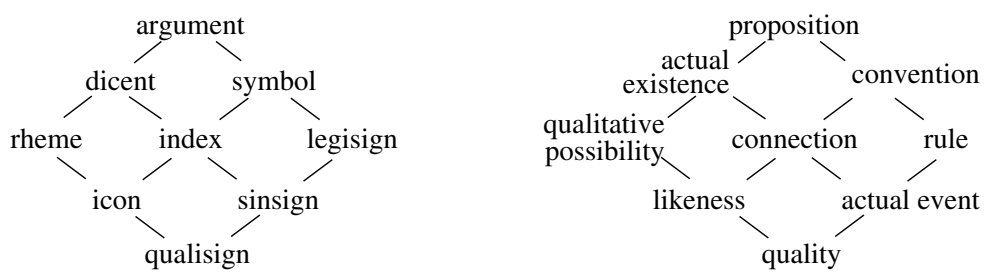

Fig. 2. The Peircean sign aspects (left) and corresponding mundane terms (right)

By virtue of the dependency between the categories, the set of sign aspects defines and induced hierarchy. Sign aspects that are higher in the hierarchy involve all lower category sign aspects. 
In [8] we have shown that the KIF process model can be assigned a Peircean semiotic interpretation. This is illustrated by the isomorphism between the diagrams in Fig. 1 and Fig. 2. An analogy between positions in the different diagrams can be explained as follows.

The input, $\left[\mathrm{q}_{2} \mathrm{q}_{1} \mathrm{C}\right]$, expressing a potential for information processing, corresponds to the qualisign sign aspect, or an interpretation of a phenomenon only as a quality. The representations obtained by sorting, $\left[\mathrm{q}_{2}\right]$ (state) and $\left[\mathrm{q}_{1}\right]$ (effect), expressing a potential for a relation, correspond to the icon and sinsign sign aspects, respectively. The icon sign aspect is an expression of a relation of constituency or likeness involved in the input state, the sinsign sign aspect an expression of a relation of simultaneity or an actual event involved in the input effect. ${ }^{2}$ An explanation of a relation between other positions of the diagrams can be found in [8]. Below we can make use of the above relation between the diagrams, in Fig. 1 and Fig. 2, by referring to a stage in information processing by its Peircean sign aspect.

By virtue of its categorical foundation, KIF theory enables a definition of uniform models of information processing in any domain. As a result, different representations can be combined into a single one by means of structural coordination. This potential of the KIF process model can be used for merging individual conceptualization processes, e.g., by the bargaining parties, into a single process. The obtained structure enables pending events to be analyzed from an informational perspective in order to reveal the type of information (and communication) necessary for their realization.

According to our process model, communication can be assigned a twofold interpretation. On the one hand, it is a final relation generated by one of the parties (cf. sender), on the other hand, it is an input quality offered for processing by the other party (cf. receiver). As, in the second case, interpretation can be in need of memory information, in order to get hold of the problem of efficient communication we introduce a conceptual memory model in the next section.

\section{Efficient Communication}

Following [8], we assume that memory information can be represented by a graph, in which nodes stand for a collection of qualities perceived in earlier observations, edges for a relation between a pair of nodes. The above model enables our definition of qualities to be refined for interactions between memory information: $\mathrm{q}_{2}$ can be defined by the qualities ${ }^{3}$ shared by the nodes responding the input stimulus, $q_{1}$ by the difference between the qualities of those nodes. Formally, for a pair of nodes, $\mathrm{n}_{1}$ and $\mathrm{n}_{2}$, triggered by the input stimulus, $\mathrm{q}_{2}:=\mathrm{n}_{1} \cap \mathrm{n}_{2}, \mathrm{q}_{1}:=\mathrm{n}_{2} \backslash \mathrm{n}_{1}$ (")" designates the operation relative difference). ${ }^{4}$ Hence $\mathrm{q}_{2}$ is an expression of information shared by observations of stimuli similar to the current one (cf. a state), $\mathrm{q}_{1}$ is an expression of a change (cf. an effect). The relation between stimulus and memory response, which can be in the sense of agreement and possibility, enables memory information to be represented as focus- and complementary-type qualities, respectively.

\footnotetext{
${ }^{2}$ Note that a state may occur in itself, an effect always assumes the existence of a state.

3 A definition of the qualities themselves is beyond the horizon of this paper.

${ }^{4}$ We assume that $\mathrm{q}_{2}$ is always defined by a non-empty collection of qualities.
} 


$$
\mathrm{n}_{1}=\{\mathrm{A}\} \bullet \mathrm{n}_{2}=\{\mathrm{A}, \mathrm{B}\}
$$

Fig. 3. Sample interpretation of memory information: $n_{1} \cap n_{2}=A$ (state), $n_{2} \backslash n_{1}=B$ (effect)

An algorithm for efficient communication can be defined as follows. We assume that the parties develop a representation of the negotiation process by themselves and by the bargaining other party, through merging (cf. Sect. 3). Communication (c) by one of the parties (sender) is offered for processing by the other party (receiver), who generates a hypothetical interpretation of $c$ from the perspective of its import in the solution of the negotiation problem. As a result, a position $(p)$ and a sign aspect is assigned to $c$, by receiver. By interpreting $c$, as an input quality, receiver may find out if, according to his representation of the negotiation process, there is an expression ${ }^{5}$ $c^{\prime}$, in position $p$ ', which is in interaction with $c$. By analyzing the relation between memory response triggered by $c$ and $c$, receiver may generate information from the perspective of the type of interaction between $p$ and $p^{\prime}$, as follows. Qualities involved by a pair of connected nodes triggered by $c$ and $c$ ', e.g., $\mathrm{n}_{1}$ and $\mathrm{n}_{2}$, respectively, can be used for the definition of a state $\left(\mathrm{n}_{1} \cap \mathrm{n}_{2}\right)$ and an effect $\left(\mathrm{n}_{1} \backslash \mathrm{n}_{2}\right)$. Information involved in the relation between this state and effect can be communicated to the other party, in the sense of difference (cf. abstraction), possibility (cf. complementation), and agreement (cf. predication). ${ }^{6}$ Qualities representing the enforced perspective by receiver may enable sender to adjust his representations in $p$ and $p$,' and this way, enhance his interpretation process.

Through communication the parties may force one another to broaden their focus, 'see' the input from a new perspective, apply their knowledge in an abductive fashion, in order to develop a solution for their common negotiation problem.

\section{A Case Study in Problem Elicitation}

In a case study at the Dutch software firm Sogeti Nederland B.V., we analysed an actual elicitation process by a team of clients [13]. In this process, three clients and a professional mediator were involved in the specification of a problem with the clients' database system. The entire elicitation process, that took 4 hours, was recorded and transcribed.

A problem with the clients' application software, 'myAssignment', is instigated in an elicitation process. The goal of myAssignment is to provide adequate information to employees, managers and client(s), about assignments and, most importantly, about communication between the participants of a project. The elicitation process was conducted in a separate room, in a usual setting, without intervention by the observer whose only task was to operate the fixed camera.

\footnotetext{
${ }^{5}$ Information by $c$ ' may have been generated by any one of the two parties ( $p$ and $p$ ' must be neighboring positions of the process model, depicted in Fig. 1). If it is generated by receiver, it can be tacit.

${ }^{6}$ If $c$ does not trigger memory, or $\mathrm{n}_{1}$ and $\mathrm{n}_{2}$ are not connected, this may trigger a generation of a request for a re-formulation of $c$ or a re-analysis of the input, by sender (cf. sorting).
} 
A sample elicitation session is displayed in Fig. 4. The goal of this session is the disclosure of missing functionalities in myAssignment. Utterances of the sample text are interpreted from the perspective of this goal. The results of the analysis are given below. The representations generated are recapitulated in Fig. 5.

1. (Pe) What, what I miss, ehh, in the current application ... is that ehh ... that

I have an overview, ehh ... of the steps that I find most logical.

(Ca) Hmm, hmm (with approval)

2. (Pe) What already happened.

(Ca) Yes.

(Pe) And ehh ... what turned out to be the result.

3. (Ca) History.

4. (Pe) Yes, history ...

(Ca) Yes.

(Pe) ... went that, that, ehh, that description of the assignment to the ehh... employee?

5. (Pe) For, I think I see a check mark of that application ...

(Ca) Yes.

6. (Pe) But I do not get a confirmation of anything, of ehh ...

(Ca) No.

(Pe) whether it, ... it has been sent.

7. (Pe) And I also do not know if it has been worked out by the employee.

(Ro) Hmm, hmm.

(Pe) Do you, ehh...?

8. (Ca) No, no I only know it because they tell me, like "Hi, I consulted and reached agreement and ehh ... that ehh ..."

9. (Ca) Yes of course, in the end you can read it off from the date of the last update, but you do not get ...

(Pe) O.K.

10. (Ca) ... an automatic mail or, or a mutation. For it is impossible to see what has been changed in the brief.

(Pe) $\mathrm{OK}$.

11. (Ca) So, I also miss the history, like ehh ... what was the initial assignment.

Fig. 4. A sample conceptualization of an elicitation session by Pe (Peter) and Ca (Caroline)

(1) logical steps:= 'icon', no overview:= 'sinsign'.

Peter admits that in the current application he is missing (cf. event) steps that he finds logical (cf. state).

(2) what already happened:= 'icon', no events and result:= 'sinsign'.

Peter refines his judgment, by paraphrasing his earlier concepts.

(3) no history:= 'legisign'.

Finally, Caroline recognizes the habitual concept of 'no history', in the instance ('no overview') and corresponding form ('logical steps') suggested by Peter.

(4) description of assignment:= 'rheme'; communication to employee:= 'index'. Similarly so, Peter recognizes an abstract concept involved in the input problem: 'description of the assignment'. He also refers to 'communication' towards the 'employee'. That concept is not further explained in this session (nor in the 
encompassing text) hence it must refer to background information. From the fact that Peter is expressing his 'doubt' in a proposition about the input problem (description of assignment to employee is lacking communication history:= 'argument') we conclude that all less developed sign aspects ${ }^{7}$ must be generated as well, such as the 'lack of a communication history': lack of communication history:= 'symbol' and, the 'description of the assignment' communicated to the employee: description of assignment to employee:= 'dicent'.

(5) check mark:= 'icon'; presence:= 'sinsign'.

Peter is justifying his conclusion, by referring to a possible 'presence' (cf. effect) of 'check marks' (cf. state), in myAssignment.

(6) confirmation:= 'index'.

Peter's doubt is related to his question about the existence of a conventional logging of 'confirmations' and, corresponding 'confirmed assignments': confirmation of assignment:= 'dicent'; lack of confirmation history:= 'symbol'.

(7) working out:= 'index'.

Peter doubts, if logging is actually 'worked out' by the employee. The appearance of this background information enables a re-evaluation of all more developed expressions: assignment worked out by employee:= 'dicent'; lack of logging history:= 'symbol', assignment by employee is not logged:= 'argument'.

(8) reaching agreement:= 'index'.

Caroline admits having the same doubts as Peter has. She points out that the employee, not the application software is providing her with information about reaching agreement with the client. According to her, a lack of logging is what is meant by 'missing communication history'. Hereby she is referring to 'reaching agreement' as a nested conceptualization process: assignment is in agreement:= 'argument', degenerately represented by a pointer: reaching agreement:= 'index', in the encompassing process of interpretation. ${ }^{8}$ By introducing a new 'index' expression, Caroline shows her interpretation of a common term involved in the generation of Peter's conclusion (note that a generation of the 'dicent' and 'symbol' positions is coordinated by the context ('index'), in the interpretation process).

(9)-(10) Caroline is further elaborating on her conceptualization of the nested process. In (9) she pinpoints: date of last update:= 'icon'; agreement:= 'sinsign'. In (10) she introduces: no automatic mail, no mutation information:= 'index'; lack of agreement information:= 'symbol' By making use of dependencies between the sign aspects and, the assumption that 'agreement' (cf. effect) must be related to an 'assignment', enables the nested conceptualization process to be completed: assignment $:=$ 'rheme'; agreement:= 'legisign'; that what is changed in the assignment: $=$ 'dicent'.

(11) myAssignment is missing history:= 'argument'.

Through a proposition of the nested process, the element of a 'no mutation information' is inherited in the index position of the encompassing conceptualization process. The appearance of this information as an index expression, this time not as a sign of doubt, but as one of a hypothesis, triggers a re-evaluation of all more developed input expressions. Assuming (11) is

\footnotetext{
${ }^{7}$ The set of sign aspects defines an induced hierarchy. See Fig. 2(a).

${ }^{8}$ Due to a lack of space, expressions of this process are omitted in Fig. 5.
} 
providing a conclusion of the conceptualization process so far, it follows that the subject of the process Caroline is referring to (although not explicitly mentioning) must be the application program itself: myAssignment:= 'rheme'; no mutation information in myAssignment:= 'dicent'; lack of mutation history:= 'symbol'. She concludes: myAssignment is missing history:= 'argument'.

(4) descr. of ass. to empl. is lacking comm. history

(6) confirmed ass. is lacking conf. history

(7) ass. to employee is not logged

(11) myAssignment is missing history.

(4) descr. of ass. to employee

(6) confirmation of ass.

(7) ass. worked out by employee

(11) no mutation info. in myAssignment dicent

(4) descr. of ass

(11) myAssignment rheme

(1) logical steps

(2) what already happened

(5) check mark icon
(4) lack of comm. history

(6) lack of conf. history

(7) lack of logging history

(10) lack of mutation history

symbol

\section{(3) no history}

legisign
(7) worked out

(8) reaching agreement (11) no mutation info index

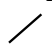

(1) no overview

(2) no events\&result

(5) presence

/ sinsign

Fig. 5. A sample conceptualization session (Peircean sign aspects are recapitulated in bold face)

The above elicitation session includes two examples of efficient communication. In (1), Peter explains his first impression of a problem with myAssignment, which he paraphrases in (2). Although he develops different icon and sinsign expressions of the input problem, e.g., 'logical steps' and 'no overview', he is unable to abstract the input and represent it from the perspective of the rheme and legisign sign aspects. Caroline, noticing Peter's problem, interprets the difference between the icon ( $c$ '='logical steps') and sinsign positions ( $c=$ '(no) overview'), and communicates this to Peter $\left(c \backslash c^{\prime}=\right.$ '(no) history'). This information, which must be familiar to Peter ('Yes, history'), although in his conceptualization process he was not able to 'see' it, enables him to realize his pending icon-sinsign event and represent the arising relation (cf. abduction) in the legisign position ('no history').

The other example, in (8), is Caroline's communication, 'reaching agreement'. In (4), Peter was able to recognize an abstract element (state) involved in the input problem ( $c$ '='description of assignment'), in the rheme position, and introduce an interpretation of this concept ( $c=$ 'description of assignment to employee'), in the dicent position. However, he was unable to express the context information involved. This refers to a potential asymmetry in Peter's interpretation process. Although he was able to conceptualize the input state from the perspectives of increasingly more meaningful sign aspects, he was unsuccessful in a similar development of representations of the input effect. Caroline's communication $(c \backslash c$ ') has a twofold contribution. It explains Peter's input representation, in the dicent position, and on top of this, it enables an interpretation of the legisign expression, 'no history', in context ('lack of logging history'). The important element in Caroline's information about a 
possible relation, 'reaching agreement' (over an 'assignment'), is coordination, between the rheme-index and index-legisign interactions, which is the essential function of representations in the index position.

\section{Summary}

Integrative negotiation can be abstracted in a process, which is isomorphic to a Peircean model of cognitive activity. The cognitive process can be analysed from an informational perspective, in order to reveal the types of information that can be necessary for a realization of the events of this process. Those types can be used for a generation of goal-driven communication increasing the efficiency of negotiation.

\section{References}

1. Fisher, R., Ury, W.: Getting to Yes: Negotiating Agreement without Giving in. Houghton Mifflin, Boston (1981)

2. Follet, M.: Constructive conflict. In: Metcalf, H., Urwick, L. (eds.) Dynamic Administration, pp. 30-49. Harpe \& Row, New York (1940)

3. Galinsky, A.D., Maddux, W.W., Gilin, D., White, J.B.: Why it pays to get inside the head of your opponent. Psychological Science 19(4), 378-394 (2008)

4. Kirk, D., Oettingen, G., Gollwitzer, M.: Mental contrasting promotes interactive bargaining. International Journal of Conflict Management 22(4), 324-341 (2011)

5. Raiffa, H.: Negotiation Analysis. Cambridge, The Belknap Press of Harvard University, Massachusetts, London (2002)

6. Sarbo, J.J.: On Well-formedness in Requirement Elicitation Processes. In: Feng, X., Liu, K., Jiang, G. (eds.) Proc. 11th Int. Conf. on Informatics and Semiotics in Organizations Beijing, China, pp. 18-25 (2009)

7. Sarbo, J.J., Farkas, J.I.: Towards meaningful information processing: A unifying representation for Peirce's signs sytems, SIGNS. Int. Journal of Semiotics 7, 1-41 (2013)

8. Sarbo, J.J., Farkas, J.I., Van Breemen, A.J.J.: Knowledge in Formation: A Computational Theory of Interpretation. Springer (2011)

9. Spanglar, B.: Integrative or Interest-based Bargaining. Beyond Intractability. In: Burgess, G., Burgess, H. (eds.) Conflict Information Consortium. University of Colorado, Boulder (2003), http: / / www. beyondintractability.org/essay/ interest-based-bargaining

10. Walton, R.E., McKersie, R.B.: A Behavioral Theory of Labor Negotiations. McGraw-Hill, New York (1965)

11. Van Breemen, A.J.J., Sarbo, J.J.: Beyond Flatland? What does semiotics add to information sciences. In: Liu, K., Li, W., Gulliver, S.R. (eds.) Proc. of the 14th Int'l Conf. on Informatics and Semiotics in Organisations, pp. 50-55. Stockholm, Sweden (2013)

12. Harnad, S.: Categorical Perception: The groundwork of cognition. Cambridge University Press, Cambridge (1987)

13. Van Breemen, A.J.J., Farkas, J.I., Sarbo, J.J.: Knowledge representation as a tool for Intelligence Augmentation. In: Igelnik, B. (ed.) Computational Modeling and Simulation of Intellect: Current State and Future Perspectives, Hershey (PA), pp. 321-341 (2011) 